\title{
Mood Disorders: Protection of the Hyper-Excited Brain, or a Risk Factor?
}

\author{
Zakaria I. Nanobashvili1,2, Irine G. Bilanishvili1', Maia G. Barbakadze1, Nadejda A. Khizanishvili1 \\ ${ }^{1}$ Department of Neurophysiology, Ivane Beritashvili Center of Experimental Biomedicine, Tbilisi, Georgia \\ ${ }^{2}$ International Caucasus University, Tbilisi, Georgia \\ Email: zaqariananobashvili@gmail.com
}

How to cite this paper: Nanobashvili, Z.I., Bilanishvili, I.G., Barbakadze, M.G. and Khizanishvili, N.A. (2019) Mood Disorders: Protection of the Hyper-Excited Brain, or a Risk Factor? Journal of Behavioral and Brain Science, 9, 54-65.

https://doi.org/10.4236/jbbs.2019.92005

Received: December 24, 2018

Accepted: February 17, 2019

Published: February 20, 2019

Copyright (c) 2019 by author(s) and Scientific Research Publishing Inc. This work is licensed under the Creative Commons Attribution International License (CC BY 4.0).

http://creativecommons.org/licenses/by/4.0/

\begin{abstract}
Emotional disturbances such as anxiety, fear, depression and aggression are often experienced by patients with temporal lobe epilepsy. These psychiatric symptoms may occur during or just after (postictal) a seizure; however, in some patients, they occur interictally (i.e. between seizures) and may profoundly change the individual's personality. Aside from confirming that a significant proportion of temporal lobe epileptics do suffer from interictal abnormal emotionality, there has been little progress to date in identifying the fundamental nature of these disturbances. There is a lack of evidences regarding the influence of activation of emotiogenic structures and emotional behavior on development of seizures. Kindling is a commonly used animal model for study of interictal emotionality and the effects of kindling and human epilepsy on emotional behavior are the primary focus of investigators and not vice versa. Respectively, the interrelation between emotional and seizure reactions was studied in Wistar albino rats. In our study we tried to elucidate: can emotional behavior evoked by stimulation of the emotiogenic zones of the hypothalamus or of induction of acute pain stress modify manifestations of generalized seizures within the period where a "full" epileptic syndrom has been stable formed earlier? Our leading hypothesis is as follow: the emotional disturbances can be considered as the emergence of instinctive behavior with an adaptive significance of defense and as a by-product of the inhibitory processes that build up to protect against the future occurrence of seizures.
\end{abstract}

\section{Keywords}

Kindling, Hypothalamus, Stress, Seizure, Rats

\section{Introduction}

Emotional disorders, such as manifestations of anxiety and fear, depression, and 
aggression, are frequently observed in patients suffering from temporal epilepsy [1] [2]. These psychiatric symptoms can arrive in the course of development of seizures or after such episodes, i.e. in a postictal mode. In some patients, however, emotional disorders are observed within intervals between seizure attacks, i.e. interictally [3]. The mechanisms responsible for susceptibility to mental disorders in subjects suffering from epilepsy remain mostly obscure.

A few clinical observations allow one to suppose that patients manifesting seizures that result from hyperactivation of the limbic structures are especially predisposed to interictal behavioral emotional disorders [4]. These structures, especially the hippocampus and amygdalar complex, are involved in the control of the emotional sphere; this is why significant changes in the functioning of the mentioned central structures can lead to mental disorders. Such functional changes can be based on injuries of the above structures or on the development of their chronic hyperexcitability [5]. On the other hand, interictal bevavioral emotional disorders were observed in epileptic patients with no structural disorders in the hippocampus or amygdalar complex. Therefore, the probability of significant interictal emotional disorders in patients with temporal epilepsy per se was confirmed. At the same time, progress in the interpretation of the fundamental nature of these disorders remains rather moderate.

Kindling is the most frequently used experimental approach for induction of temporal epilepsy [6] with the development of complex partial secondary generalization of epileptic activity. The kindling is extensively used as an animal experimental model in attempts to interpret the phenomenon of interictal emotionality and effects of epileptiform activity on emotional behavior in humans. These aspects frequently fit the main goal of the respective studies [7] [8], but the reverse statement is incorrect. It is interesting that interictal emotional disorders related to temporal epilepsy per se can be significantly modified under the action of anticonvulsants. Some agents that are effective as anticonvulsants, however, evoke noticeable side effects that can mask the interictal emotionality [9]. Moreover, some of these drugs can even intensify the interictal emotional phenomena [10].

At the same time, the corresponding emotional disorders can be interpreted as the appearance of interictal behavior possessing certain adaptive significance. They can be considered a "side product" of the inhibitory processes providing protection from the initiation of seizures in the future.

Most neuropsychiatric disorders, such as depression, mood, and anxiety disorders, are stress related disorders [11]. The stress response is a physiological reaction to environmental changes that can be positive and pro-adaptive (in most cases) or negative and maladaptive [12] [13]. Elucidation of the relations between the development of stressful state and generalization of epileptic activity remains an urgent problem of neurology. The few studies which have, thus far, attempted to analyse the influence of stress on the susceptibility of animals to convulsions have yielded conflicting results. Some authors found stressful conditions to be associated with a greater incidence and/or severity of convulsions in 
different species, including humans [14]. Others, however, have reported that stress exerts an anticonvulsant effect in epileptic gerbils and in rats and mice [15]. This variability of the influence of stress may depend on several factors such as: animal species, the agent used to elicit convulsions, the convulsive parameter considered and the characteristics of the stressogenic procedure. Anatomical structures, such as the septum, hippocampus, amygdala, raphe nucleus, and nociceptive central pathways, which are to a significant exert involved in the development of stress, participate, to a greater or smaller extent, in the initiation and spreading of epileptic phenomena in the central nervous system [16].

Despite the fact that correlations between stress and epileptic attacks have been examined in numerous experimental and clinical studies, unambiguous concepts on the nature of these relations have not been formed.

Considering this, it is expedient to use animal experimental models of epilepsy for obtaining objective information related to the above problem.

There were two main goals in our study. First, we tried to elucidate: can emotional behavior evoked by stimulation of the emotiogenic zones of the hypothalamus modify manifestations of generalized seizures within the period where a "full" epileptic syndroms has been stable formed earlier? Second, we tried to estimate changes in the ECoG and behavioral indices of seizure activity under conditions of induction of acute pain stress in rats subjected to the kindling procedure.

\section{Methods}

Wistar albino rats weighing 250 to $300 \mathrm{~g}$ were kept under conditions of a $12 / 12 \mathrm{~h}$ illumination cycle with free access to food and water. Housing of surgical manipulations with, and euthanasia of the animals were carried out in accordance with the rules and standards accepted by the scientific community of the European Union, legislation of Georgia, and the Committee on the care and use of animals in the I. Beritashvili Center of Experimental Biomedicine of Georgia. Instructions of the administration of the National Institutes of Health (Bethesda, USA) on the care and use of Laboratory animals (NIH Publication No, 88-2959) were also taken into account [17].

The animals were anesthetized by sodium pentobarbital ( $40 \mathrm{mg} / \mathrm{kg}$, i.p.). Bipolar stimulating/recording electrodes (stainless steel) were stereotaxically implanted in the ventral hippocampus and dorsomedial hypothalamus (DMH). Stereotaxic coordinates of the electrode tips in the mentioned structures were the following: 3.3 and $1.4 \mathrm{~mm}$ caudally from the bregma, 5.2 and $0.6 \mathrm{~mm}$ laterally from the sagittal line, and 6.5 and $8.5 \mathrm{~mm}$ ventrally from the brain surface, respectively [18]. A monopolar recording macroelectrode was placed on the surface of the motor cortex; the reference electrode was fixed between the skull and head muscles. The above-mentioned structures were stimulated at least 12 14 days after surgical intervention $(\mathrm{n}=12)$.

The hippocampus was stimulated using a fast kindling protocol (10-sec-long series of rectangular $400-450 \mu \mathrm{A}, 1.0 \mathrm{msec}-$ long current pulses, intraseries fre- 
quency $10 \mathrm{sec}$ ). The DMH was stimulated by 0.5 -msec-long current pulses with a $50 \mathrm{sec}$ frequency. Such stimulation evoked behavioral manifestations of fear and anxiety. For estimation of the excitability of the hippocampus and of the stability of manifestations of the epileptic syndrome, five test stimuli with 5-min-long intervals were applied to the hippocampus at different time intervals ( 2 and 4 weeks) after induction of fast kindling. Due to kindling, an epileptogenic nidus was formed in the brain of the animals, and this nidus was preserved for the entire future life period even with no additional stimulation of the hippocampus.

Then, nociceptive electrical stimulation of the fore- and hindlimbs could be applied to these animals $(\mathrm{n}=12)$ subjected to the kindling procedure. This was performed in a cage with an electrified floor (rectangular $45-\mathrm{V}$ pulses, each $15 \mathrm{sec}$ for $1 \mathrm{~min}$ ), and this resulted in the induction of acute pain stress. In animals with a preformed epileptic nidus, we recorded $(6 \mathrm{~min}, 3 \mathrm{~h}$, and $6 \mathrm{~h}$ after pain-inducing stimulation) electrocorticographic (ECoG) and behavioral manifestations of epileptic activity initiated by test stimulations of the ventral hippocampus. Behavioral and ECoG phenomena were compared with those in the absence of nociceptive stimulation. Testing under different conditions was carried out within different experimental days.

To estimate the intensity of behavioral manifestations of seizure activity, we used Racine's point scale [19]. In this scale, grade 0 corresponds to normal behavior with possible motor arrest; shaking and clonus of the facial muscles are grade 1; twitches of the head and backward inclinations of the body are grade 2; clonic contractions of the forelimb muscles are grade 3; elevations on the hindlimbs (rearing) and clonus of the forelimbs are grade 4; and elevations on the hindlimbs and falling of the animal on its back or side are grade 5 .

After cessation of the experiments, animals were injected with a lethal dose of Nembutal. Sites of localization of the tips of hippocampal and hypothalamic electrodes were coagulated (constant current 2 to $3 \mu \mathrm{A}$ was passed during $1-1.5 \mathrm{~min}$ ). The brain was taken off and fixed in a $4 \%$ paraformaldehyde solution on phosphate buffer. Localization of the electrode tips was verified in frontal slices.

\section{Data Analysis}

Differnces between the parameters of kindling-induced behavior were estimated using non-paired $t$-test or factorial dispersion analysis (ANOVA) with a post-hoc Bonferroni-Dunn test. The development of kindling manifestations was estimated using ANOVA for repeated measurements. Intergroup differences were considered to be significant at $P<0.05$.

\section{Results}

\subsection{Effects of Stimulation of DMH on the Seizure Activity}

In these experiments, we tried to estimate if combined stimulation of the hippocampus and DMH could weaken the seizure phenomena in animals with stable pre-formed manifestations of limbic epilepsy. First, the animals were subjected 
to isolated 40 hippocampal stimulations in the paradigm of fast kindling, and only then was their state tested by stimulations of the hippocampus applied simultaneously with those of DMH or without the latter. When such animals were tested by hippocampal stimulation 2 and 4 weeks after kindling initiation, they frequently responded with the development of several seizure attacks of the 4th and 5th grade. The mean duration of after discharges evoked by hippocampal test stimulation was significantly greater than in the course of five initial stimulations. In rats subjected to additional DMH stimulation, the intensity of seizures, as well as the duration and number of hippocampal after discharges, were significantly smaller, as compared with the respective indices in the group subjected to isolated hippocampal stimulation. Such a relation was observed within all time intervals (Figure 1 and Figure 2).

\subsection{Effects of Acute Pain Stress on the Seizure Activity}

We recorded changes in the seizure activity after electrical nociceptive stimulation of the limbs of rats with the preformed epileptic nidus under conditions of induction of pain stress and in the absence of the latter. As can be seen in Figure 3, 6 min after nociceptive electrical stimulation of the limbs the animals showed a clear trend toward decrease in the duration of ECoG seizure activity after control stimulations of the hippocampus, as compared with that under conditions of the absence of nociceptive stimulation. Periods of epileptiform ECoG activity within this time interval after stressogenic stimulation were, on average, $54.0 \pm$ $4.6 \mathrm{sec}$ long, while the respective value with noapplication of painful stimuli was $66.3 \pm 4.8 \mathrm{sec}$ (the difference did not reach the significance level because of considerable interindividual variability). Within the same time interval, the intensity of behavioral seizure manifestations after stressing correspond to $2.3 \pm 0.3$ grades, on average; the control index was $3.5 \pm 0.2$ grades (the difference was significant; $P<0.05$ ).

Three hours after painful electrical stimulation of the limbs, the above-described trend toward suppression of seizure activity evoked by test stimulations of the hippocampus became more clearly expressed. The mean duration of ECoG epileptiform activity in stressed animals was only $38.0 \pm \mathrm{sec}$, while this value in the absence of stress-inducing stimulation reached $80.25 \pm 5.2 \mathrm{sec}$. In other words, the effect of stress within the above time interval provided a more than twofold shortening of electrographic manifestations of epileptiform activity $(P<0.01)$. The corresponding estimates of the intensities of behavioral motor manifestations of seizure activity were $1.9 \pm 0.3$ and $3.4 \pm 0.2$ grades $(P<0.05)$. Six hours after application of stress-inducing stimulations, the effect of suppression of epileptic activity became smoothed to a considerable extent. Trends toward some decrease in the duration of ECoG epileptiform activity and expression of motor effects (convulsions) remained manifested but were extremely weak, and the corresponding values in stressed animals and in control rats were very close to each other and demonstrated no significant differences (Figure 3 and Figure 4). 


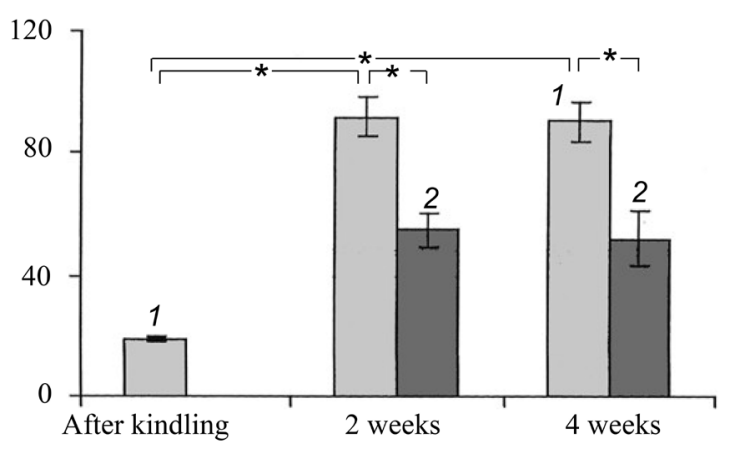

Figure 1. Effects of combined stimulation of DMH (2) on the duration (sec) of EEG seizure discharges, (ordinate) after termination of the kindling procedure.

(1) Effects of isolated test stimulation of the hippocampus.

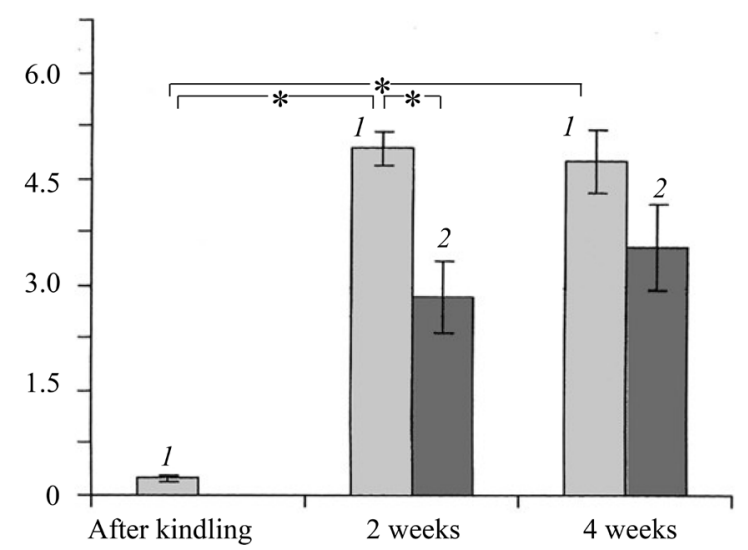

Figure 2. Effects of combined stimulation of dorsomedial hypothalamus on the intensity of behavioral seizures (ordinate), after termination of the kindling procedure. Designations are the same as in Figure 1.

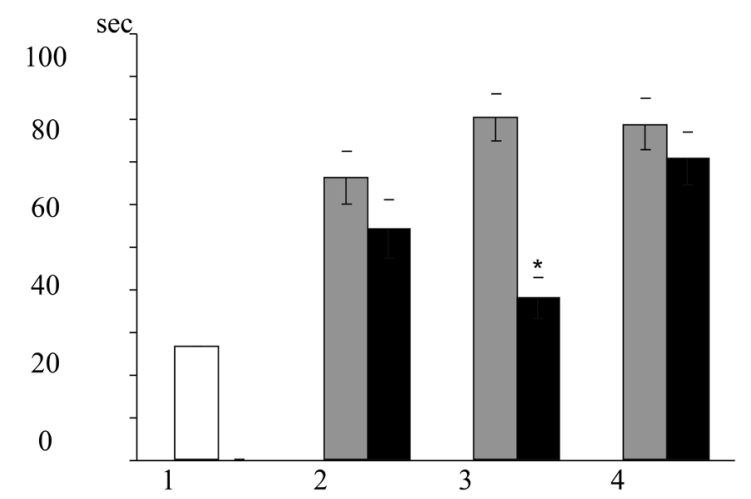

Figure 3. Suppression of electrographic seizure reactions evoked by test stimulations of the hippocampus after short-lasting stressogenic stimulation. Diagrams of the mean durations (sec) of ECoG manifestations of seizure activities in rats subjected to isolated test stimulation of the hippocampus (1) and those with kindling-induced epileptic nidus (2 - 4). Right and left columns correspond to the data obtained from animals subjected to painful electrical stimulation of the limbs ( $45 \mathrm{~V}$ each $15 \mathrm{sec}$ for $1 \mathrm{~min}$ ) and from the same animals with no such stimulation. (2 - 4) $6 \mathrm{~min}, 3 \mathrm{~h}$, and $6 \mathrm{~h}$ after nociceptive electrical stimulation of the limbs. Asteriks shown the case of significant difference between the mean values in pairs. 


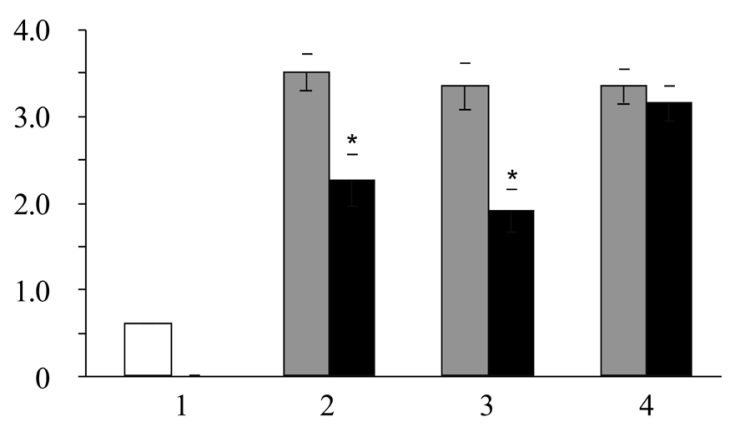

Figure 4. Suppression of behavior alseizure epileptic activity evoked by test stimulations of the hippocampus after Short-lasting stressogenic stimulation. Diagrams of the means of estimates of the intensity of epileptic motor phenomena (grades, according to Racine's point scale) Designations are similar to those in Figure 3.

\section{Discussion}

\subsection{Possible Mechanisms Changes in Seizure Activity by Stimulation of the DMH}

Our experiments for the first time gave direct proofs of the statements that activation of the DMH resulting in initiation of emotional behavior (anxiety and fear) interferes with the development of seizure activity initiated by the kindling procedure. Stimulation of the DMH significantly decreased the number and duration of generalized convulsions in rats with preliminarily evoked epileptic manifestations. Limitation of the generalization of "limbic" seizures resulting from stimulation of the hypothalamus and/or development of behavior with anxiety and fear manifestations correlates with modulation of field electrical activity of hippocampus. As is well known, the hippocampus generates rhythmic slow field activity, namely theta rhythm, after stimulation of various receptor formations and direct stimulation of a few cerebral structures [20].

Epileptic seizures frequently develop within the sleep periods. Short-term sleep is frequently used for activation of epileptiform manifestations in patients with seizure disorders [21]. As was shown earlier [22], stimulation of the hippocampus during episodes of slow-wave sleep elicits clearly manifested paroxysmal EEG discharges. At the same time, stimulation of the hippocampus with the same parameters was found to be less effective within periods of awakeness and the paradoxical sleep phase. Some authors mentioned that activation of the septo-hippocampal pathways resulting from sensory stimulation or stimulation of the hypothalamus and a few brainstem structures is accompanied by a decrease in the frequency of spiking of hippocampal pyramidal neurons [23]. On the other hand, it was shown that the frequency of discharges of hippocampal interneurons increased in this case [24]. Basket cells represent the most numerous morphological type of hippocampal interneurons; from the functional state, they are believed to be inhibitory units [25]. Precisely hippocalpal interneurons are generally qualified as theta cells. Therefore, reciprocal relations between the intensities of seizure and theta activity, on the one hand, and manifestations of seizures and the fear/anxiety state, on the other hand, can be interpreted as a sort 
of regulatory effects provided by inhibitory mechanisms under conditions of generation of the theta rhythm. Our findings allow us to suppose that augmentation of inhibitory processes in hippocampal neurons in the course of DMH stimulation can trigger mechanisms preventing the development of epileptiform activity.

The following possible mechanism could be considered as another one. The development of anxiety/fear state evoked by stimulation of the DMH can result in activation of the n. locus coeruleus (LC), and this event is capable of preventing generalization of seizure activity induced by hippocampal kindling. It is well known that the noradrenergic system of the LC is significantly involved in regulation of behavior looking like fear/anxiety [26]. Experiments with impairments or destructions of the LC substantiated the hypotheses on the anticonvulsant role of the LC noradrenergic system. The mentioned supposition was confirmed by results of experiments with LC stimulation. Activation of the neuronal system of this nucleus suppresses the initiation of seizure resulting from injections of pentylenetetrazole, kindling provided by stimulation of the amygdalar complex, and intrahippocampal injections of penicillin [27]. In turn, neurons of the LC can also activate the thalamic reticular nucleus (TRN) in the course of development of fear/anxiety behavior evoked by DMH stimulation. The TRN is richly innervated by fibers coming from the LC. Extracellular application of noradrenaline to TRN neurons or electrical stimulation of the LC results in intense excitation of neurons in the above thalamic nucleus [28]. In this respect, the following fact is rather interesting. Direct stimulation of the TRN can also suppress the development of generalized motor limbic seizures, both in the course of epileptogenesis and under conditions of the preformed epileptic state [29]. In addition to, it is well known, that high-frequency stimulation of the thalamic reticular nucleus has an anti-epileptogenic effect and is able to modify seizure synchrony and interrupt abnormal EEG recruitment of thalamo-cortical and, indirectly, corticothalamic pathways [30]. Therefore, our study demonstrated that induction of emotional behavior resulting from DMH stimulation can significantly decrease manifestations of seizure activity related to pre-formed epilepsy.

\subsection{Effect of Stress on Kindling-Induced Seizures}

Certainly the attenuation of seizure reactions by the action of stressogenic factor (electrical nociceptive stimulation) may be stipulated by the above-indicated mechanisms, though, probable mechanisms mediating the effect of acute stress on epileptic activity include, first of all, the hypothalamo-hypophyseal-adrenal axis. Since central structures of this axis are closely connected with the amygdalo-hippocampal complex, it is logical to suppose that stress-inducing factors are capable of significantly modifying the functional state of the above complex [31] [32].

In our experiments, we used electrical nociceptive stimulation of the animal's limbs, inducing the development of the state of acute stress. It seems probable that precisely the short action of pain stressogenic factors and activation of emo- 
tiogenic cerebral structures evoke augmentation of corticosterone secretion and a corresponding increase in the efficacy of GABA-ergic synaptic connections, which finally results in suppression of seizure activity. As should be believed, the level of endogenous corticosterone significantly increased within this time interval. The action of stressogenic factors also induced changes in the levels of adrenocorticotropin hormone and cortisol, and levels of these hormones depend noticeably on the type of such factors [31] [33]. The content of progesterone in the blood also increases under the influence of acute stress. As was shown, a rise in the level of this hormone exerts a modulatory effect on the activity of many cerebral neurons. In particular, a metabolite of progesterone, allopregnanolol, activates GABA-ergic receptor complexes [34]. Activation of GABA-ergic synapses inhibits generalization of seizures evoked by stimulation of the limbic structures [35].

Thus, we can conclude that the effect of an acute stressogenic factor (nociceptive stimulation) leads to a considerable decrease in the duration of ECoG epileptiform activity and partially blocks generalization of seizure reactions. The effect of the examined stressogenic factor is limited in time; the respective effects are observed for a few hours.

The emotional disturbances can be considered as the emergence of instinctive behavior with an adaptive significance of defense and as a by-product of the inhibitory processes that build up to protect against the future occurrence of seizures.

\section{Acknowledgements}

The autors thank Ms. Ninelli Skhirtladze for her help in the preparation of the manuscript.

\section{In Our Study We Tried to Elucidate}

Can emotional behavior evoked by stimulation of the emotiogenic zones of the hypothalamus or of induction of acute pain stressmodify manifestations of generalized seizures within the period where a "full" epileptic syndroms has been stable formed earlier? The emotional disorders can be interpreted as the appearance of behavior possessing certain adaptive significance. They can be considered a "side product" of the inhibitory processes providing protection from the initiation of seizures in the future.

\section{Conflicts of Interest}

The authors declare no conflicts of interest regarding the publication of this paper.

\section{References}

[1] Fisher, P.L. and Noblea, A.J. (2017) Anxiety and Depression in People with Epilepsy: The Contribution of Metacognitive Beliefs. Seizure, 50, 153-159. 
https://doi.org/10.1016/j.seizure.2017.06.012

[2] Medel-Matus, J.S., Shin, D., Sankar, R. and Mazarati, A. (2017) Kindling Epileptogenesis and Panic-Like Behavior: Their Bidirectional Connection and Contribution to Epilepsy-Associated Depression. Epilepsy \& Behavior, 77, 33-38. https://doi.org/10.1016/j.yebeh.2017.10.001

[3] Kalinchuk, L.E. (2000) Long-Term Amygdale Kindling in Rats as Model for the Study of Enterictal Emotionality in Temporal Lobe Epilepsy. Neuroscience \& Biobehavioral Reviews, 24, 691-704. https://doi.org/10.1016/S0149-7634(00)00031-2

[4] Trimble, M. (1991) Epilepsy and Behavior. Epilepsy Research, 10, 71-79. https://doi.org/10.1016/0920-1211(91)90097-Y

[5] De Lanerolle, N.C., Kim, J.H., Robbins, R.J. and Spenser, D.D. (1989) Hippocampal Interneuron Loss and Plasticity in Human Temporal Lobe Epilepsy. Brain Research, 495, 387-395. https://doi.org/10.1016/0006-8993(89)90234-5

[6] Moritomo, K., Fahnestock, M. and Racine, R. (2004) Kindling and Status Epilepticusmodels of Epilepsy. Progress in Neurobiology, 73, 1-60.

https://doi.org/10.1016/j.pneurobio.2004.03.009

[7] Adamec, R. and McKay, D. (1993) Amygdala Kindling, Anxiety and Corticotropin Releasing Factor. Physiology \& Behavior, 54, 423-431.

https://doi.org/10.1016/0031-9384(93)90230-D

[8] Waxman, S., Sand, N. and Geschwind, N. (1975) The Interictal Behavior Syndrome in Temporal Lobe Epilepsy. Archives of General Psychiatry, 32, 1580-1586. https://doi.org/10.1001/archpsyc.1975.01760300118011

[9] Engel Jr., J. (1989) Seizures and Epilepsy. PA-FA, Davis, Philadelphia.

[10] Fisher, R., Rand, J. and Kerrigan, J. (1995) Vigabatrin Toxicity. In: Lery, R., Mattson, R. and Meldrum, B., Eds., Antiepileptic Drugs, Raven Press, New York, 931-940.

[11] Musazzi, L., Tornese, P., Nathalie Sala, N. and Popoli, M. (2017) Acute or Chronic stress? A Stressful Question. Trends in Neurosciences, 40, 525-535. https://doi.org/10.1016/j.tins.2017.07.002

[12] de Kloet, E.R., Joels, M. and Holsboer, F. (2005) Stress and the Brain: From Adaptation to Disease. Nature Reviews Neuroscience, 6, 463-475. https://doi.org/10.1038/nrn1683

[13] McEwen, B.S., Bowles, N.P., Gray, J.D., Hill, M.N., Hunter, R.G., Karatsoreos, I.N. and Nasca C. (2015) Mechanisms of Stress in the Brain. Nature Neuroscience, 18, 1353-1363. https://doi.org/10.1038/nn.4086

[14] Temkin, N. and Davis, G.R. (1984) Stress as Risk Factor for Seizures among Adults with Epilepsy. Epilepsia, 25, 450-456. https://doi.org/10.1111/j.1528-1157.1984.tb03442.x

[15] Oliviero, A., Castellano, C. and Puglisi-Allegra, S. (1983) Anticonvulsant Effects of Stress: Role of Endogenous Opioids. Brain Research, 271, 193-195. https://doi.org/10.1016/0006-8993(83)91385-9

[16] Andersen, M.L., Bignotto, M., Machado, R.B. and Tufik, S. (2004) Different Stress Modalities Result in Distinct Steroid Hormone Responses by Male Rats. Brazilian Journal of Medical and Biological Research, 37, 791-797. https://doi.org/10.1590/S0100-879X2004000600003

[17] US Department of Health and Human Services (1986) Guide for the Care and Use of Laboratory Animals.

[18] Paxinos, G. and Watson, C. (1997) The Rat Brain in Stereotaxic Coordinates. Aca- 
demic Press, New York.

[19] Racine, R.J. (1972) Modification of Seizure Activity by Electrical Stimulation: II. Motor Seizures. Electroencephalography and Clinical Neurophysiology, 32, 281-294. https://doi.org/10.1016/0013-4694(72)90177-0

[20] Green, J.D. and Arduini, A.J. (1954) Hippocampal Electrical Activity in Arousal. Journal of Neurophysiology, 17, 553-557. https://doi.org/10.1152/jn.1954.17.6.533

[21] Ferraro, G., Sardo, P., Sabatino, M. and La Grutta, V. (1994) Locus Coeruleus Noradrenaline System and Focal Penicillin Hippocampal Epilepsy: Neurophysiological Study. Epilepsy Research, 19, 215-220. https://doi.org/10.1016/0920-1211(94)90064-7

[22] Buzsaki, G. and Czeh, G. (1981) Commissural and Perforant Path Interactions in the Rat Hippocampus: Field Potentials and Unitary Activity. Experimental Brain Research, 43, 429-438.

[23] Euler, V.C. and Green, J.D. (1960) Excitation, Inhibition and Rhythmic Activity in Hippocampal Cells in Rabbit. Acta Physiologica Scandinavica, 48, 110-125. https://doi.org/10.1111/j.1748-1716.1960.tb01851.x

[24] Buzsaki, G. and Eidelberg, E. (1982) Direct Afferent Excitation and Long-Term Potentiation of Hippocampal Interneurons. Journal of Neurophysiology, 48, 397-607.

[25] Freund, T.F. and Buzsaki, G. (1996) Interneurons of the Hippocampus. Hippocampus, 6, 347-470. https://doi.org/10.1002/(SICI)1098-1063(1996)6:4<347::AID-HIPO1>3.0.CO;2-I

[26] Neophytou, S.I., Aspley, U.E. and Butler, S. (2001) Effects of Lesioning Noradrenergic Neurons in the Locus Coeruleus on Conditioned and Anconditioned Aversive Behavior in the Rat. Progress in Neuro-Psychopharmacology \& Biological Psychiatry, 25, 1307-1321. https://doi.org/10.1016/S0278-5846(01)00181-6

[27] Weiss, G.K., Lewis, L. and Jimenes-Rivera, C. (1990) Antikindling Effects of LC Stimulation: Mediation by Ascending Noradrenergic Projections. Experimental Neurology, 108, 136-140. https://doi.org/10.1016/0014-4886(90)90020-S

[28] Kayama, J., Negi, T., Sugitani, M. and Iwama, K. (1982) Effects of Locus Coeruleusstimulation of Neuronal Activities of Dorsal Geniculate Nucleus and Perigeniculate Reticular Nucleus of the Rat. Neuroscience, 7, 655-666. https://doi.org/10.1016/0306-4522(82)90071-9

[29] Nanobashvili, Z., Chachua, T., Nanobashvili, A., Bilanishvili, I., Lindvall, O. and Kokaia, Z. (2003) Suppression of Limbic Seizures by Electrical Stimulation in Thalamic Reticular Nucleus. Experimental Neurology, 181, 224-230. https://doi.org/10.1016/S0014-4886(03)00045-1

[30] Pantoja-Jimenes, C., Magdaleno-Madrigal, V.M., Almazan-Alvarado, S. and Fernandez-Mas, R. (2014) Anti-Epileptic Effect of High-Frequency Stimulation in the Thalamic Reticular Nucleus on PTZ-Induced Seizures. Brain Stimulation, 7, 587-594. https://doi.org/10.1016/j.brs.2014.03.012

[31] McEwen, B.S. and Magarinos, A.M. (2001) Stress and Hippocampal Plasticity: Implications for the Pathophysiology of Affective Disorders. Human Psychopharmacology, 16, S7-S19. https://doi.org/10.1002/hup.266

[32] Taher, T.R., Salzberg, M. and Morris, M.J. (2005) Chronic Low-Dose Corticosterone Supplementation Enhances Acquired Epileptogenesis in the Rat Amygdala Kindling Model of TLE. Neuropsychopharmacology, 30, 1610-1616. https://doi.org/10.1038/sj.npp.1300709

[33] Sutula, T. and Ockuly, J. (2005) Kindling, Spontaneous Seizures, and the Conse- 
quences of Epilepsy: More than a Model. In: Pitkanen, A., Schwartzkroin, P. and Moshe, S., Eds., Models of Seizures and Epilepsy, Elsevier, Amsterdam, 395-406.

[34] Andersen, M.L., Bignotto, M., Machado, R.B. and Tufik, S. (2004) Different Stress Modalities Result in Distinct Steroid Hormone Responses by Male Rats. Brazilian Journal of Medical and Biological Research, 37, 791-797. https://doi.org/10.1590/S0100-879X2004000600003

[35] Modhaddam, B., Bolinao, M.L. and Stein-Behrens, B. (1994) Glucocorticoids Mediate the Stress-Induced Extracellular Accumulation of Glutamate. Brain Research, 655, 251-254. https://doi.org/10.1016/0006-8993(94)91622-5 